\title{
Influence of errors in determination of light lanthanides on the numerical values to indicate the light lanthanide tetrad effect
}

\author{
By Junji SHIMODA*) and Akimasa MASUDA**),+) \\ (Communicated by Kazuo YamaSAKI, M.J.A., Oct. 12, 1998)
}

\begin{abstract}
This communication reveals quantitatively the influence of experimental errors in measurement of abundances of $\mathrm{La}, \mathrm{Nd}, \mathrm{Sm}$ and Gd on the numerical index (ABEXEL) about the lanthanide tetrad effect. The resultant influence is greatest for the combinations of $\mathrm{La}(+), \mathrm{Nd}(-), \mathrm{Sm}(+)$ and $\mathrm{Gd}(-)$, and $\mathrm{La}(-), \mathrm{Nd}(+), \mathrm{Sm}(-)$ and $\mathrm{Gd}(+)$, where symbols + and - refer to positive and negative errors, respectively. If the experimental errors are assumed to be randomly $2.5 \%$, the maximum uncertainty of resulting ABEXEL turns out in general to be \pm 0.036 , and the averaged uncertainty to be \pm 0.017 . The discussions presented here are applicable to the uncertainties of the values of a system taken for normalization.
\end{abstract}

Key words : Light lanthanides; lanthanide tetrad effect; influence of experimental error; ABEXEL.

Masuda et al. ${ }^{1)}$ proposed a mathematical method to numerically indicate the degree of the lanthanide tetrad effect. When this method is applied to the data precisely determined by the stable isotope dilution technique, the indexes concerned are expressed ${ }^{2)}$ by means of ABEXEL, ABEX-3 and ABEX-4, covering La, Nd, Sm, Gd, Dy, Er, $\mathrm{Yb}$ and $\mathrm{Lu}$, usually except $\mathrm{Ce}$ and $\mathrm{Eu}$; it is assumed that ABEXEL $=$ ABEX-1 $=$ ABEX-2.

The mathematical method developed by us has been applied to water columns of the Western North Pacific, ${ }^{2-4)}$ Indian, ${ }^{5), 6)}$ and Southern ${ }^{7), 8}$ Oceans. The depth profile of ABEXEL at the Cariaco Trench, ${ }^{9), 10)}$ Venezuela, has been also studied by the same method.

In this connection, it is important to evaluate the influence of experimental errors on the resulting ABEX values. In this communication, our examination is made about the effect of measurement errors on ABEXEL values. It is noted here that the calculation of ABEXEL is made $^{1)}$ based on the abundances of $\mathrm{La}, \mathrm{Nd}, \mathrm{Sm}$ and $\mathrm{Gd}$. Although it is possible to determine the lanthanides with precisions of $1 \%$ or less in skillful determination using isotope dilution, we have provisionally presumed the

\footnotetext{
*) Department of Chemistry, The University of Electro-Communications, Chofu, Tokyo 182-8585, Japan.

**) Department of Chemistry, Faculty of Science, University of Tokyo, 7-3-1 Hongo, Bunkyo-ku, Tokyo 113-0033, Japan.

†) Correspondence should be addressed to: COLGA, 449-1 Shimo-Yasumatsu, Tokorozawa, Saitama 359-0024, Japan.
}

probable random errors of $2.5 \%$ for all of $\mathrm{La}, \mathrm{Nd}, \mathrm{Sm}$ and and Gd. There can be sixteen $\left(2^{4}\right)$ sets of combination of positive (plus) and negative (minus) errors.

In order to assure the realistic numerical values, our examination was carried out based on the data on a seawater sample at the depth of $256 \mathrm{~m}$ from the Cariaco Trench. ${ }^{10)}$ The results of our examination are presented in Table I. It can be seen that, on an absolute value, sets 6 and 9 yield the maximum errors for ABEXEL as a whole effect due to experimental errors. This is mathematically reasonable, because in evaluation of ABEXEL, two parabolic functions are applied to four elements under consideration on an assumption that the quadratic coefficient for the first tetrad involving $\mathrm{La}$ and $\mathrm{Nd}$ is equal to that for the second one involving Sm and Gd (cf. Fig. 1).

Fig. 2 shows the potential maximum uncertainty values of ABEXEL for assumed random measurement errors. As shown in Table I, the absolute maximum ABEXEL uncertainty for the presumed error of $2.5 \%$ is 0.036 . However, the average uncertainty for 16 sets turns out to be 0.0172 . When the error of $1 \%$ is presumed, the corresponding average uncertainty is evaluated to be $0.00688(=0.172 \div 2.5)$. The raw data (set S) corresponding to the seawater sample gives $A B E X E L=-0.1923$ but, as mentioned above, our present concern is the estimation of the magnitude of uncertainty resulting from the experimental errors. Furthermore, note that, in the present calculation, cases of "no errors" are not involved in combination. If the "no error" cases (zero) are involved in 


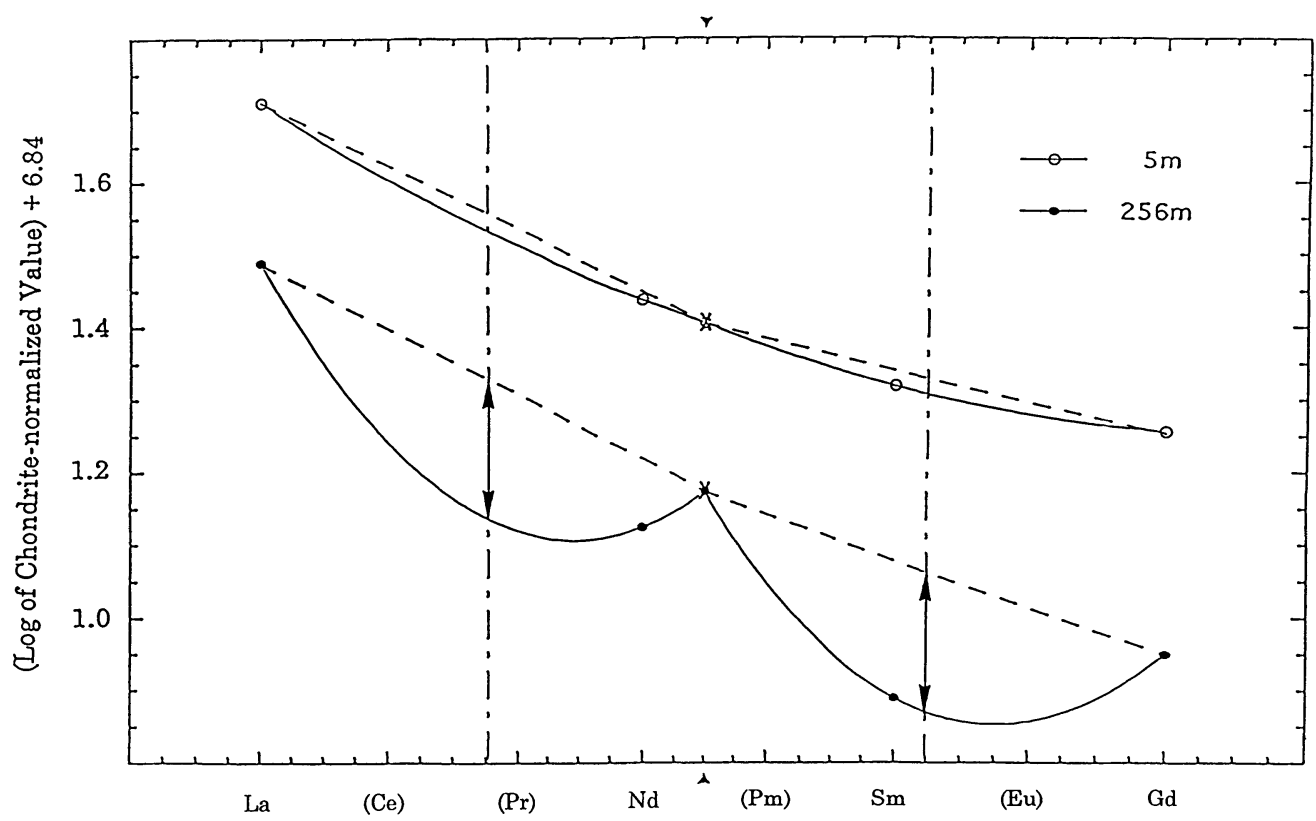

Fig. 1. Example of evaluation ${ }^{9)}$ of ABEXEL values for seawater samples at two depths of the Cariaco Trench, ${ }^{10)}$ Venezuela. Length (numerical difference between logarithms of chondrite-normalized values) of arrows for $256 \mathrm{~m}$ indicates ABEXEL value for seawater at this depth. Crosses and triangles refer to the cusp point between the first and second tetrads. The ABEXEL value for the surface water $(5 \mathrm{~m})$ is so small that arrows are not shown for this sample. Parenthesized elements are monoisotopic (Pr) or extinct (Pm), or can be anomalous in valency ( $\mathrm{Ce}$ and $\mathrm{Eu}$ ) in nature.

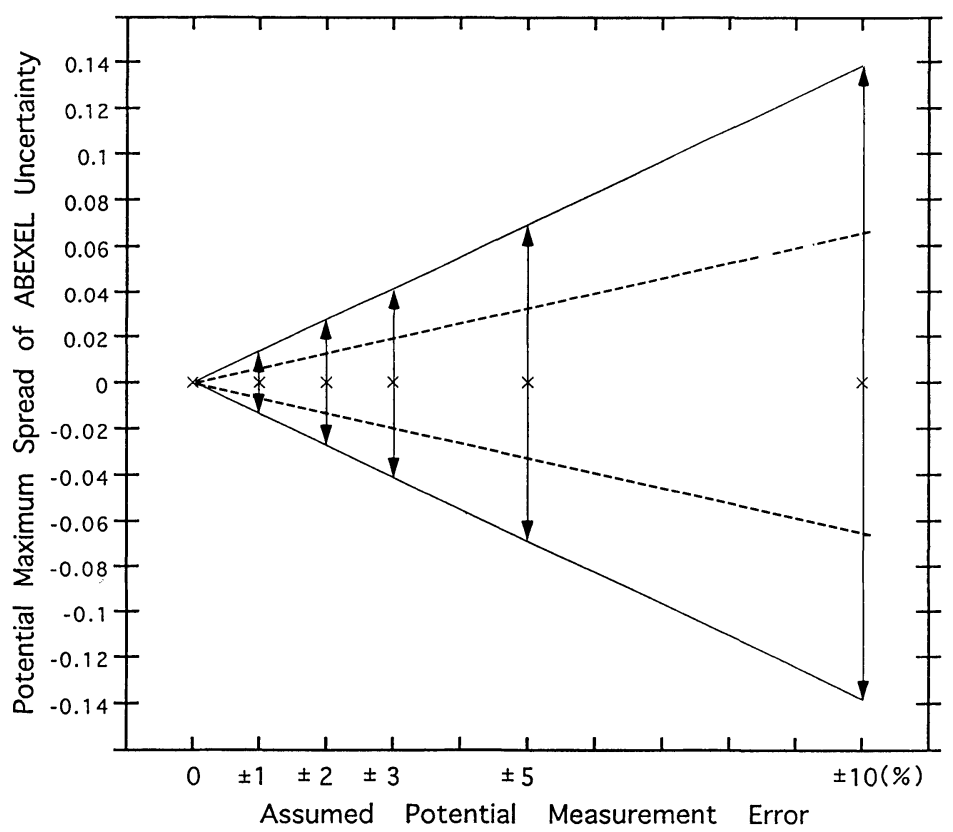

Fig. 2. Dependence of the potential maximum of ABEXEL uncertainty on assumed measurement error. Broken lines indicate the average ABEXEL uncertainty (cf. text and Table I). 
Table I. Dependence of uncertainty in calculated ABEXEL on the measurement errors*

\begin{tabular}{cccccc}
\hline $\begin{array}{c}\text { Set } \\
\text { (combined) }\end{array}$ & La & Nd & Sm & Gd & $\begin{array}{c}\text { Deviation } \\
\text { (in ABEXEL) }\end{array}$ \\
\hline S & 0 & 0 & 0 & 0 & 0 \\
1 & + & + & + & - & +0.014 \\
2 & + & + & - & + & -0.033 \\
3 & + & - & + & + & +0.022 \\
4 & - & + & + & + & -0.003 \\
5 & + & + & - & - & -0.019 \\
6 & + & - & + & - & +0.036 \\
7 & + & - & - & + & -0.011 \\
8 & - & - & + & + & +0.018 \\
9 & - & + & - & + & -0.036 \\
10 & - & + & + & - & +0.011 \\
11 & + & - & - & - & +0.003 \\
12 & - & + & - & - & -0.022 \\
13 & - & - & + & - & +0.033 \\
14 & - & - & - & + & -0.014 \\
15 & - & - & - & - & 0 \\
16 & + & + & + & + & 0 \\
\hline
\end{tabular}

*Symbols + and - correspond here to positive and negative errors of $2.5 \%$, respectively. Cases of "no error" are not involved in any set of combinations, excepting the set $\mathrm{S}$.

combination in addition to + and - , there can be 81 sets $\left(3^{4}=81\right)$. If all of these cases are included, the average uncertainties should be much lower. Accordingly, the virtual mean uncertainties should be smaller than the average uncertainties simply defined here. Thus, when the experimental errors of determination for lanthanides in the Cariaco Trench seawaters are taken ${ }^{10)}$ to be $1 \%$ or less, the virtual mean uncertainties for ABEXEL, i.e., for the difference between the logarithms (cf. Fig. 1) of chondritenormalized values corresponding to arrow tips are estimated to be considerably smaller than 0.0069 . Extent of this numerical value is too small to be shown in Fig. 1.

Finally it is noted here that someone may think that $\mathrm{Gd}$ is exceptionally high or anomalous, because $\mathrm{La}, \mathrm{Nd}$ and Sm appear to fall near a straight line (see Fig. 1). Such a view is not appropriate in view of "general" cases. ${ }^{11)-13)}$ The above appearance is merely due to the fortuitous positioning of $\mathrm{La}, \mathrm{Nd}$ and $\mathrm{Sm}$. However, one cannot rule out sometimes the effect of the probable overlapping of the octad effect on the tetrad effect.

Note added in proof. A general mathematical procedure corresponding to Table I is as follows; symbols + and - are dealt with as equivalent to 1.025 and $1 / 1.025$, respectively. Accordingly, "no error" is taken to be 1.000 .

\section{References}

1) Masuda, A., Matsuda, N., Minami, M., and Yamamoto, H. (1994) Proc. Japan Acad. 70B, 169-174.

2) Masuda, A., and Shimoda, J. (1996) Proc. Japan Acad. 72B, 202-207.

3) Masuda, A., and Shimoda, J. (1997) Proc. Japan Acad. 73B, 39-43.

4) Piepgras, D. J., and Jacobsen, S. B. (1988) Geochim. Cosmochim. Acta 52, 1373-1381.

5) Masuda, A., and Shimoda, J. (1997) Proc. Japan Acad. 73B, 187-191.

6) Bertram, C. J., and Elderfield, H. (1997) Geochim. Cosmochim. Acta 57, 1957-1986.

7) Masuda, A., and Shimoda, J. (1997) Proc. Japan Acad. 73B, 195-200.

8) German, C. R., Masuzawa, T., Greaves, M. J., Elderfield, H., and Edmond, J. M. (1995) Geochim. Cosmochim. Acta 59, 1551-1558.

9) Masuda, A., Shimoda, J., and Ikeuchi, Y. (1998) Geochem. J. 32, 275-280.

10) De Baar, H. J. W., German, C. R., Elderfield, H., and Van Gaans, P. (1988) Geochim. Cosmochim. Acta 52, 1203-1219.

11) Peppard, D. F., Mason, G. W., and Lewey, S. (1969) J. Inorg. Nucl. Chem. 31, 2271-2272.

12) Masuda, A., Shimoda, J., Matsuda, N., Lee, S.-G., and Shabani, M. B. (1995) Proc. Japan Acad. 71B, 283-287.

13) Kawabe, I., Toriumi, T., Ohta, A., and Miura, N. (1998) Geochem. J. 32, 213-229. 